\title{
AN EXTENSION OF A THEOREM OF NAGANO ON TRANSITIVE LIE ALGEBRAS
}

\author{
HÉCTOR J. SUSSMANN
}

ABSTRACT. Let $M$ be a real analytic manifold, and let $L$ be a transitive Lie algebra of real analytic vector fields on $M$. A concept of completeness is introduced for such Lie algebras. Roughly speaking, $L$ is said to be complete if the integral trajectories of vector fields in $L$ are defined "as far as $L$ permits". Examples of situations where this assumption is satisfied: (i) $L=$ a transitive Lie algebra all of whose elements are complete vector fields, and (ii) $L=$ the set $V(M)$ of all real analytic vector fields on $M$. Our main result is: if $M, M^{\prime}$ are connected manifolds, then every Lie algebra isomorphism $F: L \rightarrow L^{\prime}$ between complete transitive $L$ ie algebras of real analytic vector fields on $M, M^{\prime}$ which carries the isotropy subalgebra $L_{m}$ of a point $m$ of $M$ to the isotropy subalgebra $L_{m^{\prime}}$ of $m^{\prime} \in M^{\prime}$ is induced by a (unique) real analytic diffeomorphism $f: M \rightarrow M^{\prime}$ such that $f(m)=m^{\prime}$, provided that one of the following two conditions is satisfied: (1) $M$ and $M^{\prime}$ are simply connected, or (2) the Lie algebras $L$ and $L^{\prime}$ separate points. Nagano had proved this result for the case $L=V(M), L^{\prime}=V\left(M^{\prime}\right), M$ and $M^{\prime}$ compact.

1. Introduction. In [1], T. Nagano proved that a compact connected real analytic manifold $M$ is uniquely determined by any pair $\left(L(M), L(M)_{m}\right)$. Here $L(M)$ denotes the Lie algebra of all analytic vector fields on $M$ and, for a Lie subalgebra $L$ of $L(M)$ and a point $m \in M, L_{m}$ denotes the isotropy subalgebra of $L$ at $m$. In this note we shall extend Nagano's result by showing

(i) that, if $M$ is simply connected, the pair $\left(L(M), L(M)_{m}\right)$ can be replaced by a pair $\left(L, L_{m}\right)$, where $L$ is an arbitrary transitive subalgebra of $L(M)$, and

(ii) that the compactness of $M$ is not needed. Indeed, the assumption that $M$ is compact can be replaced by a weaker "completeness" condition. This condition is always satisfied when all the elements of $L$ are complete vector fields (in particular, it holds for arbitrary transitive $L$ if $M$ is compact), but it is also satisfied in many other cases. For instance, $L(M)$ is

Received by the editors March 30, 1973.

AMS (MOS) subject classifications (1970). Primary 32C05.

Copyright $\odot$ 1974, American Mathematical Society 
always complete, whether $M$ is compact or not. A precise statement of the condition is given in $\$ 2$. Its meaning is roughly the following: $L$ is complete if the integral curves of every $X \in L$ can be extended "as far as the algebra $L$ permits"'.

The organization of the paper is as follows. In $\$ 2$ we introduce our notations and basic definitions, including that of the completeness condition. In $\$ 3$ we state and prove our main result. In $\$ 4$ we add some additional comments, including a "local" version of our theorem in which completeness is not required.

2. Notations and basic definitions. Let $M$ be a real analytic manifold. We shall use $V(M)$ to denote the set of all real analytic vector fields on $M$. Then $V(M)$ is an infinite dimensional Lie algebra over the reals. If $L$ is a subset of $V(M)$ and if $m \in M$, then $L(m)$ denotes the set of all vectors $X(m)$, $X \in L$. A transitive subalgebra of $V(M)$ is a subalgebra $L$ such that the dimension of $L(m)$ is maximal for every $m \in M$.

If $X \in V(M), m \in M$, then $t \rightarrow X_{t}(m)$ denotes the integral curve of $X$ through $m$, i.e. the unique curve $\gamma:(a, b) \rightarrow M(a<0<b)$ such that:

(i) $\gamma(0)=m$,

(ii) $\dot{\gamma}(t)=X(\gamma(t))$ for $a<t<b$,

(iii) $y$ cannot be extended to a curve which satisfies (i) and (ii) and is defined on an interval larger than $(a, b)$.

For a given $t$, the set of all $m$ for which $X_{t}(m)$ is defined is open. If $m$ belongs to this set, then $X_{t}$ is a diffeomorphism of a neighborhood of $m$ onto a neighborhood of $X_{t}(\mathrm{~m})$. In particular, we can use the inverse of the differential of $X_{t}$ to "pull-back" tangent vectors at $X_{t}(m)$ into tangent vectors at $m$. If $v$ belongs to the tangent space of $M$ at $X_{t}(m)$, we shall use $X_{t}^{*}(v)$ to denote the corresponding tangent vector at $m$.

We are now ready to define completeness. A subalgebra $L$ of $V(M)$ is said to be complete if, for every $m \in M$ and every bounded sequence $\left\{t_{k}: k=\right.$ $1,2, \cdots\}$ of real numbers for which $X_{t_{k}}(m)$ is defined, the following holds:

(C) If the sequence $\left\{X_{t_{k}}^{*}\left(Y\left(X_{t_{k}}(m)\right)\right)\right\}$ converges for every $Y \in L$, then the sequence $\left\{X_{t_{k}}(m)\right\}$ has a limit point in $M$.

Remark. The vectors $X_{t_{k}}^{*}\left(Y\left(X_{t_{k}}(m)\right)\right)$ belong to the tangent space of $M$ at $m$. Therefore, it makes sense to talk about their convergence.

Examples. (A) If all the vector fields in $L$ are complete, then $L$ is complete. Indeed, if $\left\{t_{k}\right\}$ is a bounded sequence of reals, then it has a limit point. Since $t \rightarrow X_{t}(m)$ is continuous and defined for all $t$, it is clear that $\left\{X_{t_{k}}(m)\right\}$ has a limit point. 
(B) As a particular case of the preceding example, we note that, if $M$ is compact, then every subalgebra of $V(M)$ is complete.

(C) If $M$ is arbitrary, then the algebra $V(M)$ is complete. Indeed, let $m \in M, X \in V(M)$, and let $\left\{t_{k}\right\}$ be a sequence of reals for which $X_{t_{k}}(m)$ is defined and such that $\left\{X_{t_{k}}(m)\right\}$ has no limit point. We must show that there is a $Y \in V(M)$ such that $\left\{X_{t_{k}}^{*}\left(Y\left(X_{t_{k}}(m)\right)\right)\right\}$ does not converge. Let $\left\{u_{k}\right\}$ be an arbitrary sequence of tangent vectors at $m$ which does not converge. Let $v_{k}$ be the tangent vector at $X_{t_{k}}(m)$ whose pull-back is $u_{k}$ (i.e. $v_{k}=X_{-t_{k}}^{*}\left(u_{k}\right)$ ). Since the sequence $\left\{X_{t_{k}}(m)\right\}$ has no limit point, it follows from well-known properties of real analytic manifolds that there is $Y \in V(M)$ such that $Y\left(X_{t_{k}}(m)\right)=v_{k}$ for every $k$ (cf. Appendix).

We say that a subalgebra $L$ of $V(M)$ separates points if for every pair $m, m^{\prime}$ of points of $M$ with $m \neq m^{\prime}$ there exists $X \in L$ such that $X(m)=0$ and $X\left(m^{\prime}\right) \neq 0$.

If $L$ is a subalgebra of $V(M)$, then the set of all $X \in L$ such that $X(m)$ $=0$ is called the isotropy subalgebra of $L$ at $m$, and is denoted by $L_{m}$.

3. The main theorem.

Theorem. Let $M, M^{\prime}$ be connected real analytic manifolds. Let $L, L^{\prime}$ be transitive Lie subalgebras of $V(M), V\left(M^{\prime}\right)$. Assume that $L$ and $L^{\prime}$ are complete. Let $\Phi$ be a Lie algebra isomorphism from $L$ onto $L^{\prime}$. Assume there exist $m_{0} \in M, m_{0}^{\prime} \in M^{\prime}$ such that $\Phi\left(L_{m_{0}}\right)=L_{m_{0}^{\prime}}^{\prime}$. Assume, moreover, that either $M$ and $M^{\prime}$ are simply connected, or that the algebras $L$ and $L^{\prime}$ separate points. Then there exists one and only one diffeomorphism $\phi$ from $M$ onto $M^{\prime}$ such that every $X \in L$ corresponds under $\phi$ to $\Phi(X)$, and that $\phi\left(m_{0}\right)=m_{0}^{\prime}$.

Remark. When $L=V(M), L^{\prime}=V\left(M^{\prime}\right)$, the completeness assumption is automatically satisfied, as was pointed out in the previous section. Moreover, $V(M)$ always separates points (cf. Appendix). Therefore, our theorem contains, as a particular case, the result of Nagano (cf. [1]) in a stronger form because compactness is not required. Also, when $M$ and $M^{\prime}$ are compact, the completeness assumption is again always satisfied. Therefore, our theorem holds in this case for arbitrary transitive algebras, provided the manifolds are simply connected or the algebras separate points.

Proof of the theorem. First we note that our assumptions imply that $M$ and $M^{\prime}$ have the same dimension $n$. Let $M^{\prime \prime}$ be the manifold $M \times M^{\prime}$. Recall that the tangent space of $M^{\prime \prime}$ at $\left(m, m^{\prime}\right)$ is naturally identified to the direct sum of the tangent spaces of $M$ at $m$ and of $M^{\prime}$ at $m^{\prime}$. Therefore, if $X \in$ 
$V(M)$ and $X^{\prime} \in V\left(M^{\prime}\right)$, we can define a vector field $\left(X, X^{\prime}\right) \in V\left(M^{\prime \prime}\right)$ in an obvious way. Moreover, one has

$$
\left[\left(X, X^{\prime}\right),\left(Y, Y^{\prime}\right)\right]=\left([X, Y],\left[X^{\prime}, Y^{\prime}\right]\right) \text {. }
$$

Let $L^{\prime \prime}$ denote the set of all vector fields in $V\left(M^{\prime \prime}\right)$ that are of the form $(X, \Phi(X))$ for some $X \in L$. Then $L^{\prime \prime}$ is a Lie subalgebra of $V(M ")$.

Lemma 1. If $m \in M, m^{\prime} \in M^{\prime}$, then $\operatorname{dim} L^{\prime \prime}\left(m, m^{\prime}\right) \geq n$. Equality holds if and only if $\Phi\left(L_{m}\right)=L_{m^{\prime}}$.

Proof of the lemma. If $v$ is a tangent vector at $m$ then there is $X \in L$ such that $X(m)=v$ (because $L$ is transitive). Therefore the projection of $L^{\prime \prime}\left(m, m^{\prime}\right)$ into the tangent space of $M$ at $m$ is onto, and the first assertion follows. If $\Phi\left(L_{m}\right)=L_{m^{\prime}}^{\prime}$ then the projection is one-to-one, and $\operatorname{dim} L^{\prime \prime}\left(m, m^{\prime}\right)$ $=n$. Conversely, if this equality holds, then $X(m)=0$ implies $\Phi(X)\left(m^{\prime}\right)=0$, so that $\Phi\left(L_{m}\right) \subseteq L_{m^{\prime}}^{\prime}$, and therefore $\Phi\left(L_{m}\right)=L_{m^{\prime}}^{\prime}$.

We now return to the proof of our theorem. Let $M_{0}$ denote the maximal integral manifold of $L^{\prime \prime}$ through $\left(m_{0}, m_{0}^{\prime}\right)$ (cf. Appendix). Since $\Phi\left(L_{m_{0}}\right)=$ $L_{m_{0}^{\prime}}^{\prime}$, it follows from Lemma 1 that $M_{0}$ is $n$-dimensional, and that $\Phi\left(L_{m}\right)=$ $L_{m^{\prime}}^{\prime}$ for every $\left(m, m^{\prime}\right) \in M_{0}$. Let $\pi, \pi^{\prime}$ denote the projections of $M_{0}$ into $M, M^{\prime}$ respectively. If $\left(m, m^{\prime}\right) \in M_{0}$ then $d \pi$ and $d \pi^{\prime}$ are isomorphisms of $L^{\prime \prime}\left(m, m^{\prime}\right)$ (which is the tangent space of $M_{0}$ at $\left(m, m^{\prime}\right)$ ) onto the tangent spaces of $M$ at $m$ and of $M^{\prime}$ at $m^{\prime}$, respectively. Therefore, $\pi$ and $\pi^{\prime}$ are local diffeomorphisms.

We want to show that $\pi$ and $\pi^{\prime}$ are onto, and for this we shall use the completeness.

Lemma 2. Let $\left(m, m^{\prime}\right) \in M_{0}, X \in L$. Let $(a, b),\left(a^{\prime}, b^{\prime}\right)$ be the intervals on which the integral curves of $X$ through $m$ and of $\Phi(X)$ through $m^{\prime}$ are defined. Then $a=a^{\prime}$ and $b=b^{\prime}$.

Proof of the lemma. We shall show that $b^{\prime} \geq b$. It will follow by symmetry that $b=b^{\prime}$. When this is applied with $X$ replaced by $-X$, the conclusion that $a=a^{\prime}$ follows.

Assume that $b^{\prime}<b$. Let $\left\{t_{k}\right\}$ be a sequence such that $0 \leq t_{k}<b^{\prime}$ and that $\lim _{k \rightarrow \infty} t_{k}=b^{\prime}$. For $Y \in L$, write $Y^{\prime}$ for $\Phi(Y)$. Identify the tangent spaces of $M$ at $m$ and of $M^{\prime}$ at $m^{\prime}$ with $L / L_{m}$ and $L^{\prime} / L_{m^{\prime}}^{\prime}$ respectively. Let $F: L / L_{m} \rightarrow L^{\prime} / L_{m}^{\prime}$, be the vector space isomorphism induced by $\Phi$. Then, if $Y$ and $Z$ belong to $L$ :

$$
F([Y, Z](m))=\left[Y^{\prime}, Z^{\prime}\right]\left(m^{\prime}\right)
$$


If $Y \in L$, the function $f_{Y}: t \rightarrow X_{t}^{*}\left(Y\left(X_{t}(m)\right)\right)$ is real analytic from $(a, b)$ into $L / L_{m}$, and its derivative with respect to $t$ is $f_{[X, Y]}$. Therefore

$$
f_{Y}^{(n)}(0)=\left[X^{(n)}, Y\right](m), \quad n=0,1,2, \cdots,
$$

where $\left[X^{(0)}, Y\right]=Y,\left[X^{(n+1)}, Y\right]=\left[X,\left[X^{(n)}, Y\right]\right]$. Similarly, the function $g_{Y^{\prime}}: t \rightarrow X_{t}^{\prime *}\left(Y^{\prime}\left(X_{t}^{\prime}\left(m^{\prime}\right)\right)\right)$ is real analytic from $\left(a^{\prime}, b^{\prime}\right)$ into $L^{\prime} / L_{m^{\prime}}^{\prime}$, and its $n$th derivative at $t=0$ is $\left[X^{\prime(n)}, Y^{\prime}\right]\left(m^{\prime}\right)$. Therefore the isomorphism $F$ maps $f_{Y}^{(n)}(0)$ to $g_{Y^{\prime}}^{(n)}(0)$ for every $n$. By analyticity,

$$
F\left(X_{t}^{*}\left(Y\left(X_{t}(m)\right)\right)\right)=X_{t}^{\prime *}\left(Y^{\prime}\left(X_{t}^{\prime}\left(m^{\prime}\right)\right)\right) \text { for } 0 \leq t<b^{\prime} \text {. }
$$

Now $\lim _{k \rightarrow \infty} X_{t_{k}}^{*}\left(Y\left(X_{t_{k}}(m)\right)\right)=X_{b^{\prime}}^{*}\left(Y\left(X_{b^{\prime}}(m)\right)\right)$. Therefore the limit of $X_{t_{k}}^{\prime *}\left(Y^{\prime}\left(X_{t_{k}}^{\prime}\left(m^{\prime}\right)\right)\right)$ as $k \rightarrow \infty$ exists. Since this is true for arbitrary $Y^{\prime} \in L^{\prime}$, it follows from the completeness condition that the sequence $\left\{X_{t_{k}}^{\prime}\left(m^{\prime}\right)\right\}$ has a limit point $p$. By well-known properties of ordinary differential equations, there is $\epsilon>0$ such that $X_{t}^{\prime}(q)$ is defined for all $t \in(-\epsilon, \epsilon)$ and all $q$ in some neighborhood $U$ of $p$. Choose $k$ such that $X_{t_{k}}^{\prime}(m) \in U$ and that $b^{\prime}-t_{k}<\epsilon$. Then the integral curve of $X^{\prime}$ through $X_{t_{k}}^{\prime}(m)$ is defined on $(-\epsilon, \epsilon)$, and therefore $X_{t}^{\prime}(m)$ is defined for $0 \leq t<t_{k}+\epsilon$. Since $t_{k}+\epsilon>b^{\prime}$, this is a contradiction. Therefore, we must have $b^{\prime} \geq b$, and the lemma is proved.

The proof that $\pi$ and $\pi^{\prime}$ are onto is now easy. Let $m$ be an arbitrary point of $M$. Since $M$ is connected and $L$ is transitive, there exists a piecewise integral curve of $L$ which joins $m_{0}$ to $m$. In other words, there are vector fields $X^{1}, \cdots, X^{k}$ in $L$, numbers $t_{0}, t_{1}, \cdots, t_{k}$ such that $0=t_{0}<$ $t_{1}<\cdots<t_{k}$ and a continuous curve $\gamma:\left[0, t_{k}\right] \rightarrow M$ such that $\gamma(0)=m_{0}$, $\gamma\left(t_{k}\right)=m$ and that the restriction of $\gamma$ to $\left[t_{i-1}, t_{i}\right]$ is an integral curve of $X^{i}$ for $i=1, \cdots, k$. Let $m_{i}=\gamma\left(t_{i}\right)(i=0, \cdots, k)$. We show that there is $m_{i}^{\prime} \in M^{\prime}$ such that $\left(m_{i}, m_{i}^{\prime}\right) \in M_{0}$. If $i=0$ this is trivial. Suppose that our assertion is true for a certain $i$, then the integral curve of $X^{i+1}$ through $m_{i}$ is defined on an interval which contains $\left[0, t_{i+1}-t_{i}\right]$. By Lemma 2, the integral curve of $\Phi\left(X^{i+1}\right)$ through $m_{i}^{\prime}$ is defined on the same interval. Clearly, the mapping

$$
\delta: t \rightarrow\left(X_{t}^{i+1}\left(m_{i}\right), \Phi\left(X^{i+1}\right)_{t}\left(m_{i}^{\prime}\right)\right)
$$

is defined for $0 \leq t \leq t_{i+1}-t_{i}$. Moreover, $\delta$ is an integral curve of $\left(X^{i+1}\right.$, $\left.\Phi\left(X^{i+1}\right)\right) \in L^{\prime \prime}$. Since $\delta(0)=\left(m_{i}, m_{i}^{\prime}\right) \in M_{0}$, it follows that $\delta(t) \in M_{0}$ for all $t$ between 0 and $t_{i+1}-t_{i}$. Let $m_{i+1}^{\prime}=\Phi\left(X^{i+1}\right)_{t_{i+1}-t_{i}}\left(m_{i}^{\prime}\right)$. Then $\left(m_{i+1}\right.$, $\left.m_{i+1}^{\prime}\right)=\delta\left(t_{i+1}-t_{i}\right) \in M_{0}$. By induction, it follows that $m_{i} \in \pi\left(M_{0}\right)$ for every 
i. In particular, $m=m_{k} \in \pi\left(M_{0}\right)$. Since $m \in M$ was arbitrary, $\pi$ is onto. Since there is an obvious symmetry between $M$ and $M^{\prime}$, the same argument also shows that $\pi^{\prime}$ is onto.

We now show that $\pi$ and $\pi^{\prime}$ are covering maps. Let $m \in M$. We will find a neighborhood $U$ of $m$ which is evenly covered by $\pi$. Let $X^{1}, \cdots, X^{n}$ be members of $L$ such that $X^{1}(m), \cdots, X^{n}(m)$ are linearly independent. Choose $\epsilon>0$ so small that the mapping

$$
H:\left(t_{1}, \cdots, t_{n}\right) \rightarrow X_{t_{1}}^{1}\left(X_{t_{1}}^{2}\left(\ldots\left(X_{t_{n}}^{n}(m)\right) \ldots\right)\right)
$$

is defined for all $\left(t_{1}, \cdots, t_{n}\right)$ in the cube $C_{\epsilon}=\left\{\left(t_{1}, \cdots, t_{n}\right):-\epsilon<t_{i}<\epsilon\right.$ for $i=1, \cdots, n\}$ and is a diffeomorphism of $C_{\epsilon}$ onto a neighborhood $U$ of $m$. Let $A$ denote the set of all $m^{\prime} \in M^{\prime}$ such that $\left(m, m^{\prime}\right) \in M_{0}$. Then $A$ is nonempty because $\pi$ is onto. If $m^{\prime} \in A$, it follows from Lemma 2 that the mapping $K_{m^{\prime}}:\left(t_{1}, \cdots, t_{n}\right) \rightarrow \Phi\left(X^{1}\right)_{t_{1}}\left(\Phi\left(X^{2}\right)_{t_{2}}\left(\cdots\left(\Phi\left(X^{n}\right)_{t_{n}}\left(m^{\prime}\right)\right) \cdots\right)\right)$ is defined on $C_{\epsilon^{*}}$ Let $U\left(m^{\prime}\right)$ denote the set of all points in $M_{0}$ that are of the form $\left(p, K_{m^{\prime}} H^{-1}(p)\right)$ for some $p \in U$. The map $p \rightarrow\left(p, K_{m^{\prime}} H^{-1}(p)\right)$ is a homeomorphism of $U$ onto $U\left(m^{\prime}\right)$, whose inverse is the restriction $\pi_{m^{\prime}}$ of $\pi$ to $U\left(m^{\prime}\right)$. It follows that $U\left(m^{\prime}\right)$ is open in $M_{0}$ and that $\pi_{m^{\prime}}$ is a homeomorphism from $U\left(m^{\prime}\right)$ onto $U$. To show that $U$ is evenly covered we must prove that

(a) $U\left(m^{\prime}\right) \cap U\left(m^{\prime \prime}\right)=\varnothing$ if $m^{\prime} \neq m^{\prime \prime}$,

(b) $\pi^{-1}(U)$ is the union of the $U\left(m^{\prime}\right), m^{\prime} \in A$.

Assume that $m^{\prime} \in A, m^{\prime \prime} \in A, m^{\prime} \neq m^{\prime \prime}$, and that $U\left(m^{\prime}\right)$ and $U\left(m^{\prime \prime}\right)$ are not disjoint. Let $(p, q) \in U\left(m^{\prime}\right) \cap U\left(m^{\prime \prime}\right)$. Then $q=K_{m^{\prime}} H^{-1}(p)=K_{m^{\prime \prime}} H^{-1}(p)$.

Let $H^{-1}(p)=\left(t_{1}, \cdots, t_{n}\right)$. Then

$$
\begin{aligned}
\Phi\left(X^{1}\right)_{t_{1}}\left(\Phi\left(X^{2}\right)_{t_{2}}(\right. & \left.\left.\ldots\left(\Phi\left(X^{n}\right)_{t_{n}}\left(m^{\prime}\right)\right) \ldots\right)\right) \\
& =\Phi\left(X^{1}\right)_{t_{1}}\left(\Phi\left(X^{2}\right)_{t_{2}}\left(\ldots \Phi\left(X^{n}\right)_{t_{n}}\left(m^{\prime \prime}\right)\right) \ldots\right) .
\end{aligned}
$$

Using $n$ times the uniqueness of integral curves, it follows that $m^{\prime}=m^{\prime \prime}$, which is a contradiction. This proves statement (a).

To prove (b), let $(p, q) \in \pi^{-1}(U)$. This means that $p \in U$ and $(p, q) \in M_{0}$. Let $p=H\left(t_{1}, \cdots, t_{n}\right)$. Then $m=X_{-t_{n}}^{n}\left(\cdots X_{-t_{2}}^{2}\left(X_{-t_{1}}^{1}(p)\right) \cdot \cdot \cdot\right)$. By Lemma 2 , the point

$$
m^{\prime}=\Phi\left(X^{n}\right)_{-t_{n}}\left(\cdots \Phi\left(X^{2}\right)_{-t_{2}}\left(\Phi\left(X^{1}\right)_{-t_{1}}(q)\right) \cdots\right)
$$

exists. Moreover, it is clear that $(p, q)$ can be joined to $\left(m, m^{\prime}\right)$ by a piecewise integral curve of $L^{\prime \prime}$. Therefore $\left(m, m^{\prime}\right) \in M_{0}$. It follows that $m^{\prime} \in A$. Clearly, $q=K_{m^{\prime}}\left(t_{1}, \cdots, t_{n}\right)=K_{m^{\prime}} H^{-1}(p)$ and therefore $(p, q) \in U\left(m^{\prime}\right)$. This proves (b). 
Therefore $U$ is evenly covered by $\pi$. Since $m$ was arbitrary, it follows that $\pi$ is a covering map. The same argument shows that $\pi^{\prime}$ is a covering map.

If $M$ and $M^{\prime}$ are simply connected, it follows that $\pi$ and $\pi^{\prime}$ are oneto-one and onto. Since they are local diffeomorphisms, they are in fact diffeomorphisms of $M_{0}$ onto $M$ and $M^{\prime}$, respectively. The same conclusion can be reached without using the simple connectedness, if $L$ and $L^{\prime}$ separate points. Indeed, if $\pi$ is not one-to-one, then there are $m \in M, m^{\prime}$, $m^{\prime \prime} \in M^{\prime}$ with $m^{\prime} \neq m^{\prime \prime}$ such that $\left(m, m^{\prime}\right)$ and $\left(m, m^{\prime \prime}\right)$ belong to $M_{0}$. By Lemma 1, this implies that $\Phi\left(L_{m}\right)=L_{m^{\prime}}^{\prime}$ and $\Phi\left(L_{m}\right)=L_{m^{\prime \prime}}^{\prime}$. Thus $L_{m^{\prime}}^{\prime}=L_{m^{\prime \prime}}^{\prime}$, contradicting the assumption that $L^{\prime}$ separates points.

The conclusions of our theorem now follow. The assumptions imply that $\pi$ and $\pi^{\prime}$ are diffeomorphisms from $M_{0}$ onto $M$ and $M^{\prime}$, respectively. Define $\phi: M \rightarrow M^{\prime}$ by $\phi=\pi^{\prime} \pi^{-1}$. Then it is clear that $\phi$ has the desired properties.

The uniqueness of $\phi$ is easily proved. If $\phi$ satisfies the conditions of our theorem, then $\phi$ must carry the integral curves of every $X \in L$ into the integral curves of $\Phi(X)$, i.e.

$$
\phi\left(X_{t}(m)\right)=\Phi(X)(\phi(m)) .
$$

Since $\phi\left(m_{0}\right)$ must be $m_{0}^{\prime}$, it follows that $\phi(m)$ is uniquely determined for every $m$ that can be joined to $m_{0}$ by a piecewise integral curve of $L$. Since $L$ is transitive and $M$ is connected, $\phi$ is uniquely determined every where.

4. Additional remarks. The proof that $\pi$ and $\pi^{\prime}$ are covering maps depends only on the fact that $L$ and $L^{\prime}$ are transitive and complete. The conclusion that $\pi$ is one-to-one follows if either $M$ is simply connected or if $L '$ separates points. Therefore, the conclusion of our theorem is also true if $M$ is simply connected and $L$ separates points. This is true without any assumptions on $M^{\prime}$ and $L^{\prime}$ (other than completeness and transitivity). Moreover, a weaker form of our theorem is always true, namely: if $L$ and $L^{\prime}$ are transitive and complete, and if $\Phi$ is an isomorphism of the pairs $\left(L, L_{m}\right)$ and $\left(L^{\prime}, L_{m^{\prime}}^{\prime}\right)$ (i.e. $\Phi: L \rightarrow L^{\prime}$ is an isomorphism and $\left.\Phi\left(L_{m}\right)=L_{m^{\prime}}^{\prime}\right)$ then there exists a manifold $M_{0}$, a point $m_{0} \in M_{0}$, a transitive $L^{0} \subset V\left(M_{0}\right)$ and covering maps $\pi: M_{0} \rightarrow M, \pi^{\prime}: M_{0} \rightarrow M^{\prime}$ such that $\pi\left(m_{0}\right)=m, \pi^{\prime}\left(m_{0}\right)=m^{\prime}$ and that $\pi, \pi^{\prime}$ induce isomorphisms of the pair $\left(L^{0}, L_{m_{0}}^{0}\right)$ with $\left(L, L_{m}\right)$ and $\left(L^{\prime}, L_{m}^{\prime \prime}\right)$, respectively.

Finally, we observe that a local version of our theorem is true without any completeness assumptions: 
Let $L, L^{\prime}$ be transitive Lie algebras of analytic vector fields on the real analytic manifolds $M, M^{\prime}$. Let $\Phi: L \rightarrow L^{\prime}$ be a Lie-algebra isomorphism. Suppose $m_{0} \in M, m_{0}^{\prime} \in M^{\prime}$ are such that $\Phi\left(L_{m_{0}}\right)=L_{m_{0}^{\prime}}^{\prime}$. Then there exist neighborhoods $U$ of $m_{0}$ in $M, U^{\prime}$ of $M_{0}^{\prime}$ in $M^{\prime}$ and an analytic diffeomorphism $\phi: U \rightarrow U^{\prime}$ such that $\phi\left(m_{0}\right)=m_{0}^{\prime}$ and that, for every $X \in L$, the restrictions of $X$ to $U$ and to $\Phi(X)$ to $U^{\prime}$ correspond under $\phi$.

The proof of the preceding statement is very easy. Define $M^{\prime \prime}, L^{\prime \prime}, M_{0}$, $\pi, \pi^{\prime}$ as in the proof of our main theorem. It is still true that $\pi$ and $\pi^{\prime}$ are local diffeomorphisms. Let $U^{\prime \prime}$ be a neighborhood of $\left(m_{0}, m_{0}^{\prime}\right)$ which is mapped diffeomorphically by $\pi, \pi^{\prime}$ onto neighborhoods $U, U^{\prime}$ of $m_{0}, m_{0}^{\prime}$ respectively. Define $\phi: U \rightarrow U^{\prime}$ by $\phi \pi^{\prime} \pi^{-1}$. Then $\phi$ satisfies the desired conditions.

Appendix. We state here two facts that have been used in $\$ 2$ and $\$ 3$.

(A) Let $M$ be a real analytic manifold. Let $A$ be a subset of $M$ that has no limit points. For each $m \in A$ let $v_{m}$ be a tangent vector at $m$. Then there exists $X \in V(M)$ such that $X(m)=v_{m}$ for every $m \in A$.

This is a well-known property of real analytic manifolds (cf. for instance Shiga [2]).

(B) Let $M$ be a real analytic manifold, and let $L$ be a Lie subalgebra of $V(M)$. Then through every $m \in M$ there passes a unique maximal integral manifold of $L$.

Recall that an integral manifold of $L$ is a connected submanifold $S$ of $M$ whose tangent space at $s$ is $L(s)$ for every $s \in S$. An integral manifold is maximal if every integral manifold $S^{\prime}$ which intersects $S$ is in fact an open submanifold of $S$. Statement (B) is proved by Nagano in [1].

Acknowledgments. The author wishes to thank D. L. Elliott, A. Krener and W. Xotus for helpful discussions.

\section{REFERENCES}

1. T. Nagano, Linear differential systems with singularities and an application to transitive Lie algebras, J. Math. Soc. Japan 18 (1966), 398-404. MR 33 \#8005.

2. K. Shiga, Some aspects of real analytic manifolds and differentiable manifolds, J. Math. Soc. Japan 16 (1964), 128-142; ibid. 17 (1965), 216-217. MR 30 \#2517; 31 \#6243.

DEPARTMENT OF MATHEMATICS, RUTGERS UNIVERSITY, NEW BRUNSWICK, NEW JERSEY 08903 